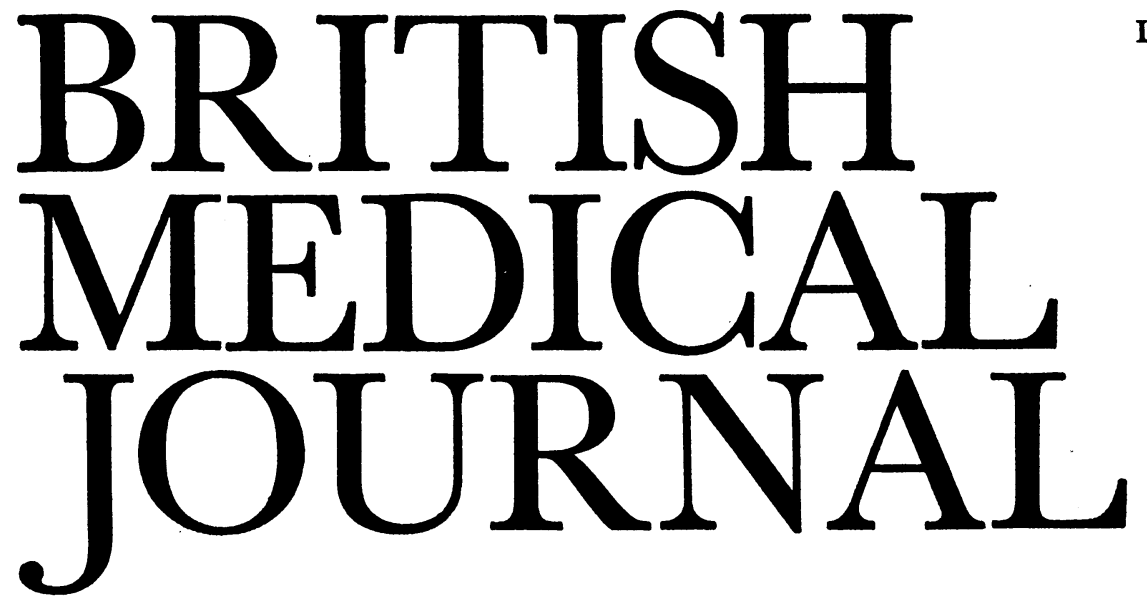

\title{
Diet and Coronary Disease
}

Coronary artery disease presents us with a problem of alarming proportions. It is already the major cause of death in most affluent communities, and its incidence, particularly among young and middle-aged men, appears to be increasing. Unfortunately despite the immense amount of money which has been spent on research in this field we still do not know the cause of the condition or how to prevent it.

Many people with raised levels of plasma cholesterol or triglyceride or both are particularly at risk for the development of coronary artery disease. 1 Though the epidemiological evidence for the association is convincing, there is no proof that the reduction of serum lipids leads to a corresponding reduction in risk of myocardial ischaemia. Even so most physicians attempt to lower serum lipid levels when they are clearly abnormal, using drugs such as cholestyramine and clofibrate and modification of the diet. By contrast few doctors would advise their other patients to take measures to lower their serum lipids despite evidence that the risk of coronary heart disease is proportional to serum cholesterol and triglyceride levels, even when these are within the "normal" range. 23

It seems fairly certain that much coronary artery disease is preventable, because environmental factors are of greater significance in determining its incidence than racial characteristics. ${ }^{4}$ Dietary habits seem to be particularly important, and impressive epidemiological evidence links the number of deaths from coronary artery disease in different countries to the amount of saturated fat which is eaten. 56 The effect of a high intake of saturated fat, derived mainly from dairy products and fat meats, may be mediated through an effect on serum cholesterol levels, because these fall when the intake of saturated fat is reduced even if there is an equicaloric substitution of polyunsaturated fat, derived largely from vegetable sources.

High plasma cholesterol levels are clearly linked with premature development of coronary atheroma, but the increased incidence of coronary heart disease which is associated with a high intake of saturated fat may not depend on increased formation of atheromatous plaques. Many who have studied the matter believe that recent increases in the incidence of coronary artery disease cannot be explained by a corresponding increase in atherosclerosis, and it is clear that other factors must play a part. ${ }^{4}$ As there is no direct evidence in man that the atheromatous process is reversible, it is often argued that there is little point in advocating tedious dietary restrictions for middle-aged people if coronary artery disease is well advanced. If, however, the clinical disease is dependent on additional, more easily reversible factors, then there would be grounds for optimism when advocating dietary change.

Regardless of the mechanisms involved it is clearly crucial that we should establish whether a change of diet can lead to a reduction in the risk of coronary artery disease. Unfortunately many problems arise in the design and interpretation of appropriate trials. In those which have been conducted the experimental diets were so designed as to reduce serum cholesterol levels: saturated fats were low and polyunsatured fats high. The results of several of the most important trials were reviewed by a committee convened by the National Heart Foundation of New Zealand.4 Its admirable report concluded, though cautiously, that a favourable clinical effect seemed to be apparent when the experimental diet was continued for at least two years. But it pointed out that studies so far have been done only on older people. The critical experiment of altering the dietary fat intake of children and young adults has not yet been undertaken.

What advice then should we give to our patients and what advice may be offered to the general population? It is far from proved that changes in diet, particularly in later life, prevent or modify coronary artery disease. Even so the Boards of Health in Finland, Norway, and Sweden, the Inter-Society Commission for Heart Disease Resources in the U.S.A., ${ }^{7}$ and Committees of the National Heart Foundations of Australia ${ }^{8}$ and New Zealand ${ }^{4}$ have all seen fit to make strong recommendations to the public. Their main recommendations were similar and include, firstly, a reduction in calorie intake to achieve and maintain optimal weight; secondly, a decrease in dietary cholesterol; and, thirdly, a substantial reduction in dietary saturated fat. To 
maintain body weight the reduction in saturated fat must be countered by another source of calories. An increase in carbohydrates might itself increase the risk of coronary artery disease by increasing production in the liver of triglyceriderich lipoproteins ${ }^{9}$ of very low density. This can be avoided by substituting polyunsaturated for saturated fat in the diet. To facilitate such dietary change the Committee of the New Zealand Heart Foundation ${ }^{4}$ advocated that the composition of foodstuffs should be widely publicized and that those which contain polyunsaturated oils should have no restrictions placed on their availability. S. Dayton and colleagues $^{10}$ reported that the incidence of carcinoma was increased in a group of people on a diet high in polyunsaturated fat, but this observation does not appear to have been substantiated in other studies. ${ }^{11}$ Certainly the New Zealand committee saw no reason for restricting the consumption of polyunsaturated fats even though it did not consider there was any particular need to promote their use in the whole population.

While it would seem sensible to endorse these recommendations there is clearly not enough evidence to justify a full-scale campaign aimed at changing the dietary habits of the nation. ${ }^{12}$ But the accumulating evidence is impressive, and it seems likely that if it is clearly presented many people will wish to modify their diet. Though cigarette sales have not fallen precipitously, it is now clear that many other sensible people besides doctors have given up the smoking habit. To such people the fat on their bacon could become just as unnecessary as the smoke from their cigarettes.

1 Rifkind, B. M., British fournal of Hospital Medicine, 1970, 4, 683. 2 Kannel, W. B., Castelli, W. P., Gordon, T., and McNamara, P. M., Annals of Internal Medicine, 1971, 74, 1 .

3 Carlson, L. A., and Böttiger, L. E., Lancet, 1972, 1, 865.

4 National Heart Foundation of New Zealand, Coronary Heart Disease -A New Zealand Report. Dunedin. John McIndoe Ltd., 1971. 5 Keys, A., Circulation, 1970, 41, 42, Supplement 1 .

- Morris, J. N., and Gardner, M. J., American fournal of Medicine,

Morris, J. N., and Gardner, M. J., American fournal of
$1969,46,674$.

B National Heart Foundation of Australia, Medical fournal of Austialia, 1971, 1, 1155.

- Fredrickson, D. S., Levy, R. I., and Lees. R. S., New England fournal of Medicine, 1967, 276, 34.

10 Dayston, S., et. al., Lancet, 1968, 2, 1060.

11 Ederer, F., Leren, P. Turpeinen, O., and Frantz, I. D., Lancet, $1971,2,203$.

12 Fredrickson, D. S., British Medical fournal, 1971, 2, 187.

\section{Presymptomatic Detection of Huntington's Chorea}

Huntington's chorea is inherited in an autosomal dominant fashion. Symptoms usually begin after the age of 30 , which means that people who develop the disease usually have already conceived their offspring by the time it first appears. The child of an affected parent who has seen the gradual progression of choreo-athetoid movements and mental deterioration to a totally crippled state and death is all too well aware that he himself has a $50 \%$ chance of developing the disease. Suicidal depression is not unusual in this group of people. If some effective method were available for detecting the gene for Huntington's chorea before the development of signs and symptoms, it would at least be possible to reassure half of these patients who are at risk.

There are indications that such a test may now be possible. Two groups of workers have recently reported on the use of levodopa in the investigation of people genetically at risk of developing the disease. ${ }^{1-3}$ The basis of the test is that patients with Parkinson's disease who are treated with levodopa in high dosage frequently develop dyskinesias, including choreo-athetoid movements very similar to those seen in Huntington's chorea. It is possible that Huntington's chorea is due to an abnormal reaction to dopamine. It is therefore also posible that such movements may. be induced by levodopa in asymptomatic persons who are later to develop the disease. In a recent report ${ }^{3}$ patients and controls received levodopa in gradually increasing doses to a maximum of $2.5 \mathrm{~g}$ daily for a total of 10 weeks. In a second part of the study levodopa at a dose of $800 \mathrm{mg} /$ day was given in combination with a peripheral dopa decarboxylase inhibitor. The age range studied was from 17 to 33 years. None of the 24 controls developed dyskinetic movements on either regimen. However, about a third of the people genetically at risk of developing Huntington's chorea did develop such dyskinetic movements.

Many things need to be elucidated before the levodopa test can be accepted as an effective method for the detection of presymptomatic Huntington's chorea. Though false positive reactions appear to be unlikely, a larger series of normal persons will be required to make certain that an occasional normal person may not develop dyskinetic movements during this test. Moreover, it still remains to be proved that the people who develop dyskinetic movements will in fact go on to the full development of Huntington's chorea. Though they seem likely to do so, a long follow-up of these positive reactors is awaited with interest. Finally, a negative response in a person genetically at risk of developing the disease cannot be regarded as proof of the absence of the gene for it. Again a long follow-up will be required to find out the proportion of false negative responses and at what period in the life of the patient a false negative response can be expected to occur.

Until these questions have been answered it will be difficult to know exactly what to tell the patient. In fact it has been discussed whether it is ethical even to carry out such investigations until an effective treatment for Huntington's chorea is available. ${ }^{4}$ At present the only reasonable way in which the results of the test may be applied is in advising individuals on the risk of transmitting the disease to children. Many people seek advice on whether there is any sign of their having the disease before they embark on a family of their own. If the reaction to levodopa is positive, it is probably reasonable to explain that there is a possibility that the disease might be passed on to the children even though there is no certainty that the individual himself may develop it. This is the sort of advice that many of them are seeking.

\footnotetext{
Klawans, H. L., Paulson, G. W., and Barbeau, A., Lancet, 1970, 2, 1185

Cawein, M., and Turney, F., New England fournal of Medicine, $1971,284,504$

Klawans, H. L.i Paulson, G. W., Ringel, S. P., and Barbeau, A., 4 Gaylin, W., New England fournal of Medicine, 1972, 28\%, 1361.
}

\section{Papovaviruses and Human Disease}

The papovaviruses are small icosahedral (20-sided) viruses which have until recently seemed to be of only limited interest in clinical medicine. The group includes the various papilloma or wart viruses of man and animals, polyoma 